\title{
México: La educación en salud reproductiva del adolescente debe fortalecerse
}

Frontiers in Reproductive Health

Follow this and additional works at: https://knowledgecommons.popcouncil.org/departments_sbsr-rh How does access to this work benefit you? Let us know!

\section{Recommended Citation}

"México: La educación en salud reproductiva del adolescente debe fortalecerse," FRONTERAS Resumen de Investigación Operativa. Ciudad de México: Population Council, 2003. 


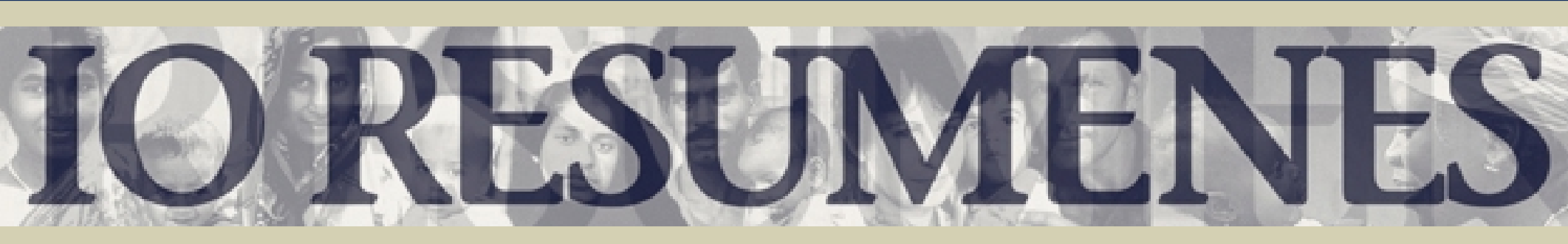

México

Adolescentes

\section{La educación en salud reproductiva del adolescente se fortalece}

\section{Investigación operativa}

Resumen 22

\begin{abstract}
Los jóvenes en México tienen conocimientos generales sobre salud reproductiva, pero su escasa comprensión de la fisiología de la reproducción y de las infecciones de transmisión sexual los hace vulnerables a las consecuencias de comportamientos de riesgo. Se recomienda que los directores de los programas sigan fortaleciendo la educación en salud reproductiva del adolescente, tanto para los jóvenes como para los proveedores de servicios.
\end{abstract}

\section{Antecedentes}

El sexo sin protección entre los jóvenes en México con frecuencia ocasiona embarazos no planeados y nacimientos no deseados, y los expone a contraer infecciones de transmisión sexual (ITS), entre ellas el VIH/SIDA; estas consecuencias afectan la vida y las oportunidades de chicas $\mathrm{y}$ chicos por igual.

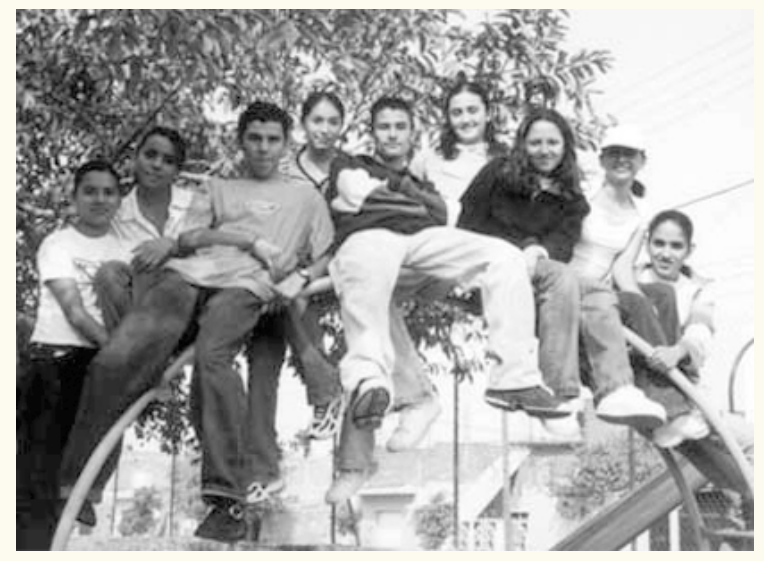

De 1999 a 2002, FRONTERAS trabajó con la organización no gubernamental Mexfam para estudiar la viabilidad, eficacia y costo de realizar intervenciones en los ámbitos comunitario, clínico y escolar para mejorar la salud reproductiva de los jóvenes de 10 a 19 años de edad. Se implementaron intervenciones en ocho ciudades a través del Programa Gente Joven (PGJ) de Mexfam, y cuatro ciudades más sirvieron como grupo de control. Los investigadores trabajaron con los coordinadores y promotores del PGJ - miembros de la comunidad como maestros, líderes comunitarios, proveedores de salud, y grupos de pares_- quienes asistieron a cursos sobre salud reproductiva del adolescente y posteriormente los impartieron. En la intervención comunitaria, los promotores establecieron contacto con individuos y con grupos a través de obras de teatro, eventos deportivos, desfiles y conciertos. Dentro del componente clínico, los proveedores ofrecieron servicios amigables para jóvenes. En cuatro de las ciudades de la intervención se implementó también un componente escolar, dentro del cual los maestros y los médicos escolares capacitados dieron pláticas y realizaron otras actividades para fortalecer los programas existentes de educación sexual.

\section{Hallazgos}

- Estudiantes, padres y maestros, más de 14,000 en total, asistieron a los cursos y pláticas impartidos por los coordinadores y promotores del PGJ. La mayoría de los adolescentes (cerca del 70\%) refirió haber recibido educación sexual en la escuela. Los 100 proveedores participantes informaron que habían prestado más de 60,000 servicios médicos a adolescentes, más de la mitad de ellos relacionados con la salud reproductiva.

- El conocimiento sobre anticoncepción, que era alto, aumentó con el tiempo. La proporción de jóvenes con nociones sobre planificación familiar, las cuales abarcaban alrededor de seis métodos anticonceptivos tanto en la línea de base como en la línea final, aumentó del 57 al 66 por ciento. El conocimiento sobre cómo utilizar correctamente un método fue mixto; por ejemplo, sólo el 20 por ciento de los adolescentes sabía que la píldora debe tomarse todos los días. Casi todos los adolescentes que saben algo sobre anticoncepción conocen el condón. Más del 80 por ciento tenía algún conocimiento sobre el uso correcto del mismo. 
El conocimiento acerca del VIH/SIDA se incrementó del 63 al 85 por ciento; en todas las ciudades, aún en las del grupo de control, se registró un incremento similar. Entre las personas que habían oído hablar sobre el VIH/SIDA, el 83 por ciento mencionó las relaciones sexuales y las transfusiones sanguíneas como medios de contraer la infección. El conocimiento de que los condones son un medio de protección aumentó del 56 al 78 por ciento. Menos del 5 por ciento de los jóvenes entrevistados mencionó la fidelidad como una forma de prevenir el VIH. Además, menos de la mitad de los adolescentes entrevistados después de la intervención habían oído hablar acerca de otras infecciones de transmisión sexual.

- En general, se registra poca actividad sexual. De los jóvenes de 10 a 19 años de edad, sólo el 10 por ciento había tenido una experiencia sexual cuando se realizó la encuesta de línea de base (y el 11\% en la línea final) y el 43 por ciento de los adolescentes que ya habían tenido una experiencia sexual estaban casados o viviendo con su pareja. Casi todos iniciaron la actividad sexual después de los 14 años. En todos los grupos, tanto antes como después de la intervención, los hombres tenían el doble de probabilidades que las mujeres de utilizar un método (ver el siguiente cuadro). La gran mayoría obtuvieron su métodocon mayor frecuencia un condón-en una farmacia (el $85 \%$ ) y no en un centro de salud.

Experiencia sexual en jóvenes de 10 a 19 años, sitios de la intervención y de control, 2002

\begin{tabular}{|c|c|c|c|c|}
\hline \multirow[b]{2}{*}{ Han tenido rel. sexual } & \multicolumn{2}{|c|}{$\begin{array}{cc}\text { Intervención combinada } \\
\begin{array}{c}\text { Hombres } \\
\text { (n=591) }\end{array} & \begin{array}{c}\text { Mujeres } \\
(n=663)\end{array}\end{array}$} & \multicolumn{2}{|c|}{$\begin{array}{l}\text { Control (\%) } \\
\text { Combres Mujeres } \\
(n=309) \quad(n=352)\end{array}$} \\
\hline & 11 & 7 & 13 & 11 \\
\hline Edad de la primera vez & 15.7 & 15.8 & 15.6 & 16.1 \\
\hline Utilizó método la primer & a vez & 21 & 50 & 22 \\
\hline
\end{tabular}

- Una comparación entre las áreas de la intervención y las de control mostró pocos cambios en el comportamiento que pudieran atribuirse a la intervención. Esto posiblemente se debió a las grandes áreas de influencia, a la contaminación en la implementación de la intervención, o a factores sociales que tal vez afectaron las actitudes y los comportamientos.

Aunque ocurrieron cambios positivos tanto en el grupo de control como en las áreas experimentales, el análisis demostró que el conocimiento sobre métodos anticonceptivos y el uso de servicios de salud reproductiva eran significativamente mayores entre los jóvenes que habían estado directamente expuestos a las intervenciones del PGJ.

- Los costos marginales fueron similares en las dos áreas en las que se llevó a cabo la intervención: cerca de US\$235,000. El componente escolar fue costoso debido a los altos costos no financieros de cerca de US\$100,000 (como el tiempo del personal y la reasignación de sus tareas). Las actividades comunitarias tuvieron el costo financiero más alto (entre US $\$ 40,000$ y $\$ 60,000$ ), lo cual refleja las variadas escalas y frecuencia de las actividades.

\section{Utilización}

- Mexfam ha adoptado varias de las estrategias administrativas que se introdujeron durante el proyecto, entre ellas, un nuevo sistema de información enfocado en la recolección de información para la toma de decisiones de manera local, nuevos criterios de selección y procedimientos de seguimiento para los voluntarios, y un enfoque innovador en el diseño de los programas para jóvenes.

\section{Implicaciones normativas}

Las intervenciones para mejorar el acceso de los jóvenes a los servicios de salud reproductiva deben desarrollar esquemas de capacitación integral para proveedores en los que se incluyan metas de servicio e indicadores para medir los avances. También se recomienda desarrollar programas dirigidos a los farmaceutas como importantes proveedores de salud para los jóvenes.

Septiembre 2003

\section{Fuente:}

Vernon, Ricardo y Maricela Durá. 2004. "Improving the reproductive health of youth in Mexico [Mejorar la salud reproductiva de los jóvenes en México]," FRONTIERS Final Report. Washington, DC: Population Council. Disponible en nuestro portal: http://www.popcouncil.org/pdfs/frontiers/FR FinalReports/Mex Youth.pdf o por correo electrónico: frontiers@pcdc.org

Este proyecto se llevó a cabo con el apoyo de la AGENCIA DE LOS ESTADOS UNIDOS PARA EL DESARROLLO INTERNACIONAL (USAID) bajo el Acuerdo de Cooperación No. HRN-A-00-98-00012-00. 